Beate Henn-Memmesheimer (Hg.)

\title{
Sprachliche Varianz \\ als Ergebnis von \\ Handlungswahl
}

Sonderdruck aus RGL 198

Max Niemeyer Verlag Tübingen 1998 


\section{Beate Henn-Memmesheimer/Ute Bärnert-Fürst/Anke Denzer/ \\ Heike Gallery}

\section{Nonstandard als Faktor bei der Strukturierung kommunikativer Situationen}

Zur charakteristischen Verteilung von Indikatoren und Markern

\section{Indikatoren und Markierungen}

Im Blick der Dialektologen sind Nonstandardmuster Indikatoren für regionale Herkunft. Sie werden faßbar in wissenschaftlichen Untersuchungen und, so weit die Sprecher selbst soziale Kategorisierungen und Stereotype daraus ableiteten, in entsprechenden Alltagsdiskursen, in Ortsspott und partiell in Dialektzensus. Soziolinguistische Analysen fokussieren Zusammenhänge zwischen Nonstandardverwendungen und wissenschaftlich konstruierten Sozialstatus, bzw. sozialen Zuschreibungen, die Sprecher in Befragungen explizit machen (,Jemand, der so spricht, kann nur Putzfrau sein.'). Ethnographische Analysen zeigen ebenfalls soziale Kategorisienungen auf, die mit Nonstandard verbunden sind - aber nur solche, die von Sprechern spontan für relevant gehalten werden (,Jugendlicher", „die feinen Leute“, „Asos“, „Wildsäue“, „die Ausländer“" "). Nonstandard- oder Standardsprechen wird also einerseits als fixes Merkmal, als Indikator ${ }^{2}$, wahrgenommen und in einem Feld von sozialen Kategorien und Stereotypen lokalisiert.

Andererseits bewegen sich die von uns untersuchten Sprecher virtuos zwischen Standard und Nonstandard. Es zeigt sich in verschiedenen Arbeiten der letzten Jah$\mathrm{re}^{3}$, daß zumindest ein Teil der von Sprechern verwendeten Nonstandardmuster als „Marker ${ }^{* 4}$, d.h. funktional verwendet wird. Allerdings greifen die bisherigen Darstellungen jeweils nur vereinzelte Episoden aus Texten heraus. Am Beispiel zweier Handlungskonstellationen eines Gesprächs soll im folgenden gezeigt werden, daß die Sprecher Nonstandardvarianten bzw. Standardvarianten nicht so benutzen, als gehörten sie zu verschiedenen Systemen und wären cum grano salis in das je andere System übersetzbar, sondern daß die Sprecher die Varianten zu sich ergänzenden Teilen ihres Repertoires machen und sie mit so verschiedenen Signifikanzen beladen, daß man - nach den von ihnen konstituierten Verwendungsweisen -

Kategorien nach, Kallmeyer 1994, 444ff. Vgl. auch Hundt, 1992.

Vgl. unten Abschnitt 6.1

Auer 1986, Schlobinski 1987.

Vgl. Leuenberger/Hofer in diesem Band, Abschn. 2.3 
manches nur mit Standardmustern, anderes nur noch mit Nonstandardmustern formulieren kann.

\section{Exkurs: Die Diskrepanz zwischen expliziter Bewertung und vielfältiger Verwendbarkeit von Nonstandardmustern}

Nonstandardmuster wurden bei einer Befragung ${ }^{5}$ im pfälzischen und kurpfälzischen Raum noch 1996 explizit mit Ländlichkeit und niedrigem Bildungsstand, mit defizitärer Sprachkompetenz und Zugehörigkeit zu einer unteren sozialen Schicht verbunden, wie es die schulischen Erfahrungen, die legitimierten Lehrpläne und wissenschaftliche Untersuchungen aus den 60er und 70er Jahren nahelegen. Die seit den 80er Jahren beschriebenen spezifischen Funktionen des Nonstandard in offiziellen Situationen, in regionalistisch orientierten Texten der Politik, Konsumwerbung und Literatur und die sympathisch wirkenden Akzente wie das Honoratiorenschwäbisch von Theodor $\mathrm{Heu}^{6}{ }^{6}$ blieben marginale Erscheinungen. Daß regionalsprachliche Akzente als argumentativ wirksam oder angenehm empfunden werden, scheint nicht verallgemeinerbar zu sein, sondern an das Gesamtbild einer Person gebunden.

De facto werden aber Nonstandardmuster häufig verwendet. Diese Inkonsistenz läßt sich auflösen, indem man zwischen langfristigen Einstellungen und situativen Bewertungen unterscheidet. Die in der Sozialpsychologie uneinheitlich verwendeten Termini Einstellung und Bewertung werden dabei auseinander dividiert: Einstellungen werden als latente Wissensstrukturen verstanden. Sie sind stereotyp und beziehen sich auf die soziale, regionale und domänenbezogene Verteilung von Standard und Nonstandard. Bewertungen dagegen sind situationsabhängig und kommen erst dann zum Tragen, wenn es eine Situation erfordert, das heißt, wenn ein Sprecher Varianten der Sprache einsetzt oder diesen Einsatz reflektiert. Unter expliziten Bewertungen sind konkret geäußerte Meinungen gegenüber bestimmten Objekten zu verstehen. Implizite Bewertungen zeigen sich im tatsächlichen Umgang mit sprachlichen Formen. Nonstandardformen werden im Kontext von Interviews explizit meist negativ bewertet. Ursache sind hier wahrscheinlich einerseits die stereotypen Einstellungen und andererseits die interviewspezifische Tendenz zu politisch korrekten Antworten sowie die Zustimmungstendenz ${ }^{7}$. Die tatsächliche, kontextuell vielfältig variierende Verwendung läßt jedoch auf implizit differenziertere Bewertungen schließen, die sich bezeichnenderweise bisweilen auch in offenen, die Situationen stärker thematisierenden Interviews ${ }^{8}$ zeigt. Die Bewertung und damit die Wahl der Nonstandardmuster ergibt sich offenbar aus allen soziologisch

\footnotetext{
Dissertationsprojekt von Anke Denzer, Veröffentlichung voraussichtlich 1998.

Vgl. Sitta 1980, 211.

Esser 1990, 232.

Macha 1991, Ziegler 1996, 249 u.a..
} 
relevanten Merkmalen der Kommunikationssituation und den je verschiedenen kommunikativen Zwecken ${ }^{9}$. In ein komplexeres Modell der Bewertung sollte zusätzlich eine Kosten-Nutzen-Abwägung einbezogen werden: Für manche Sprecher ist standardnahes Sprechen mit erheblich mehr Aufwand verbunden als für andere je nach individueller Lerngeschichte.

\section{Materialbasis}

Das untersuchte Schlichtungsgespräch „Die Mopeds“ ist Teil des Korpus „Institutionelle Konfliktgespräche“ des Instituts für deutsche Sprache, Mannheim. Es wurde im kurpfälzischen Raum im Zusammenhang mit einer Evaluierung der Institution Schlichtung aufgenommen ${ }^{10}$ und wird derzeit ediert ${ }^{11}$ Die Transkription mußte im Rahmen unserer Fragestellungen verändert werden; es waren eine enge literarische Umschrift - der übersichtlichen Lesbarkeit halber - und eine phonetische Transkription - der computativen Auswertung wegen - nötig. ${ }^{12}$ Das Gespräch dauert 45 Minuten, es sind vier Personen beteiligt: Herr Heilmann ${ }^{13}$ (Antragsteller) will die ständigen verbalen Entgleisungen seines langjährigen Nachbarn, Herm Boos, (Antragsgegner), nicht mehr hinnehmen und schaltet daraufhin einen Anwalt ein, der ein Schlichtungsverfahren vor einem Schlichter der Vergleichsbehörde einleitet.

\section{Die kommunale Vergleichsbehörde}

\subsection{Institutionelle Situationsdefinition}

Die kommunale Vergleichsbehörde in Baden-Württemberg ist eine staatliche Institution der Rechtsprechung, die als Vorstufe der Gerichte angesehen werden kann. Ihre Aufgabe besteht darin, einen bestehenden Konflikt zwischen Konfliktparteien in einem förmlichen vor- bzw. außergerichtlichen Verfahren zu schlichten. Schlichten ist somit ein rechtliches Verfahren zur Bearbeitung oder im besten Fall

9 Vgl. dazu u.a. die Unterscheidung zwischen objektiver Situation und subjektivem Handlungskontext, wie sie Fazio 1990 trifft; und vgl. das Problem der „Rahmung" einer Situation, wie

${ }_{10}$ es u.a. bei Esser 1996 gefaßt ist.

10 Zur wissenschatlichen Begleitung und Bedeutung der Vergleichsbehörde in Baden-Württemberg und dem Schiedsmannswesen in Nordrhein-Westfalen siehe u.a. Röhl (Hrg.) 1987 und Klein 1995.

11 Schröder 1996.

12 Die Aufbereitung des Materials wurde von Barbara Bernasko und Christine Gerbich unterstützt. Die literarische Umschrift wurde in wesentlichen Teilen von Anke Denzer und Anke Langendörfer erarbeitet. Bezüglich der Transkriptionsregeln sei hier nur kurz darauf verwiesen, daß die Orthographie der Morpheme/Lexeme immer dann beibehalten wurde, wenn die

13 Orthoepie realisiert wurde.

3 Personennamen und geographische Namen sind fingiert. 
zur Lösung sozialer Konflikte. ${ }^{14}$ Vorsitzender einer Vergleichsbehörde (Schlichter) kann entweder der Bürgermeister der Gemeinde oder ein Bediensteter der Gemeindeverwaltung sein. Dies ist in der Regel ein Sachbearbeiter mit juristischen Kenntnissen, der formal unter der Fachaufsicht eines Richters des zuständigen Amtsgerichts steht. Vergleichsverhandlungen wie „Die Mopeds" unterliegen also einem gerichtlichen Paradigma. Dieser institutionelle Rahmen strukturiert die jeweilige konkrete Schlichtungssituation maßgeblich und weist dem Gespräch und den beteiligten Akteuren unveränderbare Merkmale zu. ${ }^{15}$

Innerhalb dieser Rahmenbedingungen stellen die Schlichtungsgespräche für die beteiligten Akteure unterschiedliche Situationen dar. Für den Schlichter sind Vergleichsverhandlungen bekannte Situationen, in denen er aufgrund seiner Stellung als Vorsitzender auf ein etabliertes Handlungsschema und auf eigene Erfahrungen zurückgreifen kann. Für die beiden Konfliktparteien ist die Vergleichsverhandlung in der Regel eine unbekannte Situation, mit der sie vorher nicht konfrontiert waren. Auch wenn das Handlungsschema als kulturell verbreitet gilt, ist davon auszugehen, daß die Konfliktparteien eher vage Vorstellungen vom Ablauf eines Schlichtungsgesprächs haben. ${ }^{16}$

\subsection{Die Personen und die erwartbaren Ziele und Interessen}

Jeder Vergleichsverhandlung liegt ein Streitfall zugrunde, den die beteiligten Parteien auf privater Basis nicht kooperativ zu lösen vermochten, so daß die Positionen der Beteiligten als Antragsteller (A), Antragsgegner (B), Schlichter (C) und Protokollführer klar definiert sind; gelegentlich tritt darüber hinaus ein Anwalt einer Konfliktpartei $\left(A_{1}\right.$ oder $\left.B_{1}\right)$ auf.

Der Schlichter verfolgt als vorrangiges Ziel die gütliche Einigung zwischen den Konfliktparteien. Als Grundlage für die gütliche Einigung verfolgt der Schlichter das Zwischenziel, eine Konfliktversion ausfindig zu machen, die beiden Parteien gerecht wird. Da sich der Antragsteller in irgendeiner Art und Weise geschädigt fühlt, wird er versuchen, so viele Kompensationsforderungen wie möglich durchzusetzen. Im Gegensatz dazu wird der Antragsgegner das Ziel verfolgen, sich drohenden Sanktionen zu entziehen. ${ }^{17}$

\footnotetext{
14 Vgl. Klein 1996.

16 Vgl. Klein und Nothdurft 1987.

Zur Terminologie bekannte/unbekannte Situationen vgl. Essers Unterscheidung definierter 17 und nicht-definierter Situationen 1976 und 1996. Vgl. Klein und Nothdurft 1987.
} 


\begin{tabular}{ll|}
\hline Schlichter: & Auffinden einer gemeinsam akzeptierten Konfliktversion \\
& Herbeiführung einer gütlichen Einigung \\
Antragsteller: & Kompensationsforderungen/Sanktionen \\
Antragsgegner: & Vermeidung von Sanktionen \\
\hline
\end{tabular}

Abb. 4.1: Erwartbare Ziele und Interessen innerhalb der Vergleichsverhandlung

\subsection{Die Handlungsschritte der Akteure}

Im idealtypischen Ablauf von Schlichtungsgesprächen sind nach Nothdurft und Klein 1987 folgende Handlungsschritte erwartbar:

$$
\begin{array}{ll}
\text { 1. Herstellung der Schlichtungssituation } & \text { 3. Herbeiführung einer Einigung } \\
& - \text { Entwicklung eines Einigungsvor- } \\
\text { 2. Rekonstruktion des Konflikts } & \text { schlags } \\
\text { - Etablierung der Anschuldigung } & - \text { Ratifizierung des Einigungsvor- } \\
\text { - Stellungnahme } & \text { schlags } \\
\text { - Konfliktaushandlung } & \\
& \text { 4. Auflösung der Schlichtungssituation }
\end{array}
$$

Abb. 4.2: Idealtypische Ablauffigur (Klein \& Nothdurft, 1987)

Im Kontext einer Beschreibung der institutionellen Situation ist diese idealtypische Ablauffigur so weiterzuentwickeln, daß sie eine stringente Analyse der Handlungsschritte differenziert nach Akteuren erlaubt. Argumentativ stellt es beispielsweise einen Unterschied dar, welche Konfliktpartei sich zu dem Konflikt äußert, so daß wir eine Unterscheidung bezüglich der Konfliktdarstellung des Antragstellers (A) und der Konfliktgegendarstellung des Antragsgegners (B) getroffen haben. Diese Ausdifferenzierung ermöglicht es, einzelne Handlungsschritte als für Schlichtungsgespräche konstitutiv und aus der institutionellen Funktion der jeweiligen Akteure ableitbar darzustellen. Im folgenden Schema sind die Handlungsschritte aufgelistet (graphisch durch Spiegelstriche markiert) und zu Handlungskonstellationen (graphisch durch Fettdruck markiert) zusammengefaßt.

Diese Handlungsschritte werden in Gesprächen nicht streng getrennt in fester zeitlicher Abfolge hervorgebracht. Zwar obliegen die Herstellung der Schlichtungssituationen institutionell definiert dem Schlichter, aber das heißt nicht, daß in dieser Konstellation nur der Schlichter spricht. Es gibt in allen Teilen des Gesprächs Interventionen (kurze Einwürfe) der jeweils nicht handlungstragenden/handlungsfortführenden Sprecher. 
Herstellung der Schlichtungssituation

- Eröffnung (C)

- Funktionserläuterung (C)

Rekonstruktion des Konflikts

Etablierung der Anschuldigung

- Schriftsatzvorstellung (C)

Konfliktaushandlung

- Aufforderung zur Konfliktdarstellung (C)

- Konfliktdarstellung (A)

- Sicherstellung der Konfliktdarstellung (C)

- Aufforderung zur Konfliktgegendarstellung $(C)$

- Konfliktgegendarstellung (B)

- Sicherstellung der Konfliktgegendarstellung (C)

- Konfliktformulierungsangebot (C)

- Aufforderung zur Stellungnahme zur Vorgeschichte/Folgegeschichte (C)

- Rekonstruktion der Vorgeschichte/ Folgegeschichte (A, B)

- Sicherstellung der Vorgeschichte/ Folgegeschichte (C)

- organisierende Interventionen (C)

- Bewertung des Konflikts (C)

Herbeiführung einer Einigung Entwicklung eines Einigungsvorschlags

- Einigungsvorschlag formulieren (C)

- Aufforderung zur Stellungnahme zum Einigungsvorschlag (C)
- Stellungnahme zum Einigungsvorschlag (A, B)

- Modifizierung des Einigungsvorschlags (C)

- Aufzeigen der juristischen Konsequenzen bei Nichteinigung $(C)$

- Aufzeigen von bisherigen Gemeinsamkeiten (C)

- Vertrauen in zukünftiges Verhalten herstellen (C)

- Aufzeigen unrealisierter Konfliktlösungen im Vorfeld (C)

- Aufzeigen langfristiger Konfliktlösungen (C)

- Erklärung langfristiger Konfliktlösungsbereitschaft $(A, B)$

\section{Kostenfrage}

- Klärung der Kostenregelung (C)

- Aufforderung zur Stellungnahme zur Zahlungsweise (C)

- Stellungnahme zur Zahlungsweise (A, B)

Ratifizierung des Einigungsvorschlags

- Protokoll (C)

- Auffordenung zur Stellungnahme zum Protokoll (C)

- Stellungnahme zum Protokoll (A, B)

- Sicherstellung des Protokolls (C)

Auflösung der Schlichtungssituation

- Unterzeichnung des Protokolls

Abb. 4.3: Die Handlungskonstellationen mit den nach Akteuren differenzierten Handlungsschritten in der Vergleichsverhandlung 
5. Situationsstrukturierung und Nonstandardverwendung Hypothese

Wenn dem Gebrauch von Standard- und Nonstandardmustern situationsstrukturierende Funktion zugeschrieben werden kann, dann müßten in funktional (institutionell) unterschiedlichen Handlungskonstellationen unterschiedliche Standard- und Nonstandardvorkommen nachzuweisen sein. Der Text wird auf zwei Ebenen analysiert:

1. inhaltlich, nach Handlungskonstellationen

2. linguistisch, nach Dichte der Nonstandardmuster.

Es sollte sich dann zeigen, daß der Schlichter sich erstens an diesen Handlungskonstellationen orientiert, und zweitens in seine Handlungen, die die Schlichtung voranbringen, signifikant Standard-/Nonstandardbewegungen einbezieht. Wir werden dies an zwei markanten Handlungskonstellationen exemplifizieren.

\section{Die Sprache}

6.1 Laut- und Morphemspektren des Schlichters: Repertoire mit Indikatoren und Markern

Ausgangspunkt der Analyse ist die individuelle Variationsbreite von Standard bis Nonstandard, die ein Sprecher aufweist: das Repertoire. Darauf werden seine variierenden Standard- oder Nonstandardverwendungen bezogen und als mehr oder weniger standard- oder nonstandardorientiert beschreibbar.

Wir ermitteln die Variationsbreite eines Sprechers für jeweils ein Gespräch, bzw. für die Gespräche, an denen er beteiligt ist (vgl. Tabellen). Bei der Beschreibung der Standard- und Nonstandardmuster werden diejenigen Muster als Standard gesetzt, die kodifiziert und durch Bildungsinstitutionen legitimiert sind. ${ }^{18}$ Die Rekonstruktion von Lautspektren geht von Morphemen aus, die in zwei oder mehr lautlichen Varianten realisiert werden, von denen mindestens eine als Standard, die anderen als Nonstandard gelten. Z.B. sind für das Morphem da die Varianten [da], $\left[\mathrm{d} \mathrm{\Lambda}\right.$ ] und [do], für das Morphem fest die Varianten [fest] und [fe $\int \mathrm{t}$ ], für das Morphem -lich- die Varianten [-liç-], [-liç-] und [-lif-] belegbar. Lautliche Varianten haben per definitionem keinen Phonemstatus. Einige der Spektren sind wahrscheinlich kontinuierlich. Dies ließe sich durch beliebig enge lautschriftliche Wiedergabe anzeigen. Die in manchen Fällen auftretende Schwierigkeit zu unterscheiden, ob es sich noch um den Standardlaut oder schon um den Nonstandardlaut

18 Zur Definition von Standard- und Nonstandardmustern siehe Henn-Memmesheimer 1986 und 1996. 
handelt, bzw. welcher der Nonstandardlaute vorliegt, spricht jedenfalls für die Annahme von Kontinua in einigen Spektren.

Auch wenn sich Varianten als Orte auf einem Spektrum beschreiben lassen, ist die Reihung kein Maß für „Dialekttiefe“ (wie etwa in Ammon 1973): es gibt z.B. Regionen, die im grundständigen Dialekt [ $]$ haben und solche, die [c] haben

\begin{tabular}{|c|c|c|}
\hline $\begin{array}{l}\text { Beispiele aus den Äußerungen des Schlichters, } \\
\text { generalisiert über das ganze Gespräch }\end{array}$ & $\begin{array}{l}\text { Rekonstruierte } \\
\text { Spektren }\end{array}$ & $\begin{array}{l}\text { Schema der rea- } \\
\text { lisierten Berei- } \\
\text { che der Spektren }\end{array}$ \\
\hline StdV-....--NstdV & StdV-----NstdV & StdV----NstdV \\
\hline Indikatoren & & \\
\hline$[/]-----[-v e r s-](06 / 10)$ & {$[z]-\ldots---[s]$} & \\
\hline$[/]---[-r I \varphi d-](17 / 08),\left[-r I \int d-\right](17 / 03)$ & {$[c]---[\zeta]---\left[\int\right]$} & \\
\hline Marker & & \\
\hline$[\mathrm{dan}](01 / 23) \cdots-\cdots[\mathrm{d} \wedge \mathrm{n}](01 / 20),[\mathrm{d} \cap n](10 / 34)$ & {$[\mathrm{a}]---[\Lambda]---[\supset]$} & + \\
\hline [ain- $](02 / 11)----[$ ain- $](23 / 28),[\supset \mathrm{i}-](21 / 9)$ & {$[\mathrm{ai}]---[\Lambda \mathrm{i}]---[\supset \mathrm{i}]$} & \\
\hline$[\operatorname{aux}](22 / 08)-\cdots--[\wedge u x](17 / 05)$ & {$[a u]-\cdots--[\Lambda u]$} & \\
\hline$[a n-](14 / 13)-\cdots--[\Lambda n-](17 / 07),[a ̃-](13 / 25)$ & {$[a]---[\Lambda]---[\tilde{a}]$} & \\
\hline [auf](12/02)----[nuf ](04/28), [uf](14/18) & {$[\mathrm{au}]--[\Lambda U]--[U]$} & \\
\hline$[$ main-](05/33)---[m^in-](13/08), [me:n-](11/02) & {$[\mathrm{ai}]--[\Lambda \mathrm{Ai}]---[\varepsilon]$} & \\
\hline [tsvai](13/09)----[tsve:](11/18) & [ai]-_-_e] & \\
\hline$[\mathrm{da}-](02 / 09)-----[\mathrm{d} \partial-](03 / 07)$ & {$[a]-\ldots[--\ldots]$} & \\
\hline$[$ vi:r-](06/07)----[vee-](12/13) & {$[i:]-\cdots[\varepsilon]$} & +-on- + \\
\hline [mYs-](18/31)----[mIs-](12/12) & {$[Y] \cdots[I]$} & \\
\hline$[\mathrm{k} \curvearrowright \mathrm{m}-](01 / 23)---[\mathrm{kum}-](13 / 07)$ & {$[0]-\ldots[u]$} & $+\cdots$ \\
\hline$[k œ n-](01 / 11)----[k e n-](21 / 05)$ & {$[\mathfrak{e}]-\cdots$} & $+\cdots$ \\
\hline [pasa-](04/28)----[basə-] $(12 / 02)$ & $[p] \cdots-\ldots b]$ & \\
\hline [part-](01/09)--.-[pard-](12/09) & {$[t]-\ldots[d]$} & 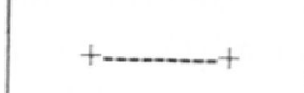 \\
\hline$[1 \mathrm{Y}: \mathrm{k}-](13 / 06)-\cdots--[1 \mathrm{l}: \mathrm{g}-](13 / 07)$ & {$[\mathrm{k}] \cdots-\ldots$} & $+\ldots+$ \\
\hline$[$ ha:b-](12/04)----[hav-](12/14) & {$[b]-\ldots$} & +.-- \\
\hline [sa:g-](02/09)------[sa:x-](02/11) & {$[g]-\ldots[x]$} & 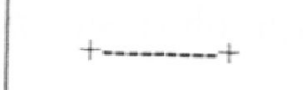 \\
\hline$[-\mathrm{i}: \mathrm{ig}](01 / 20)---\left[-1 \int-\right](12 / 22)$ & $[g]-\ldots-n]$ & $+\ldots+$ \\
\hline
\end{tabular}


$[$ vered-](05/21)---[ver-](12/12)
$[$ Andər-](05/24)---[anər-](08/10)

\begin{tabular}{|c|c|}
\hline [ed]--------[r] & +--_-_-_-_+ \\
\hline [nd]--- & +--_- \\
\hline
\end{tabular}

Die Ziffer vor dem Schrägstrich gibt die Seite, die nach dem Schrägstrich die Zeile des Tran= kripts an.

StdV

NstdV

-

Tab. 6.1:
Standardvariante

Nonstandardvariante

nur die Nonstandardvarianten sind belegt

das gesamte Spektrum ist belegt

Lautkontinua des Schlichters, generalisiert über das gesamte Schlichtungsgespräch
„Die Mopeds"

Die Tabelle stellt die Lautspektren dar, die der Schlichter im Gespräch einsetzt. Mit Ausnahme der Zwischenlaute in [a - $\Lambda$ - o], [ai - $\Lambda \mathrm{i}-$ ji] und $\left[\varsigma-\epsilon-\int\right]$ sind alle anderen Nonstandardvarianten in der dialektologischen oder soziolinguistischen Literatur beschrieben. ${ }^{19}$

Die dritte Spalte der Tabelle visualisiert schematisch das Fehlen verschiedener Standardlaute. Die als Standard kodifizierten Laute, stimmhaftes [z] und [c], kommen im beschriebenen Repertoire nicht vor. All diese Nonstandardlaute, zu denen wir über das gesamte Schlichtungsgespräch hinweg keine Standardvarianten ausmachen konnten, nennen wir in der Tradition von Labov Indikatoren, die variierten nennen wir Marker $^{20}$.

Darüber hinaus sind für den Schlichter Variationen verschiedener Flexionsmorpheme belegbar. Infinitive beispielsweise werden entweder mit dem Standardmorphem [-(ə)n] oder nonstandardorientiert mit [-ə] markiert, Partizipia II werden vor [s] oder [ $\left.\int\right]$ mit dem Standardmorphem [gə-] oder nonstandardorientiert mit [g-] markiert. ${ }^{21}$ Diese Spektren sind diskontinuierlich. Tabelle 6.2 gibt einen Überblick.

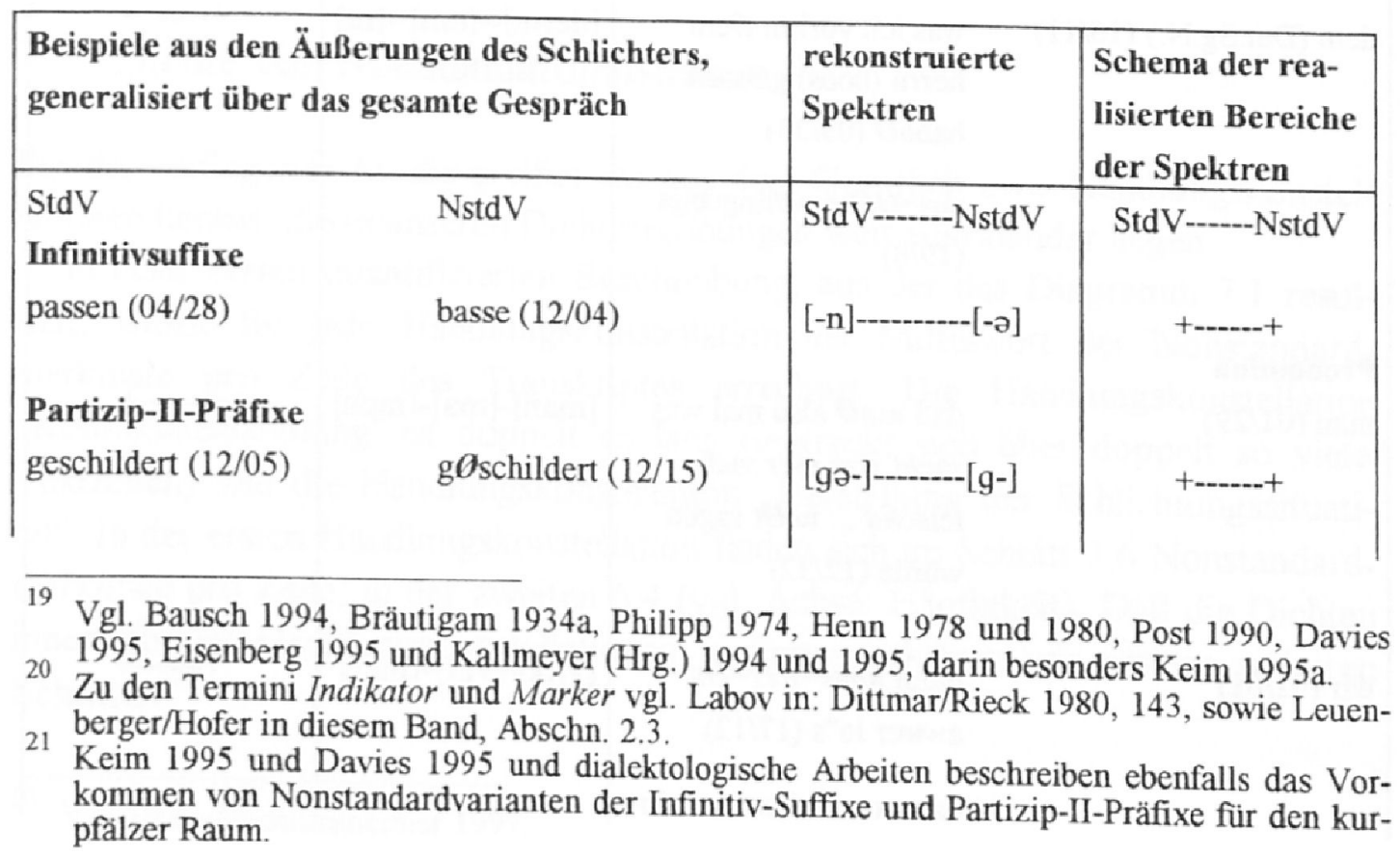




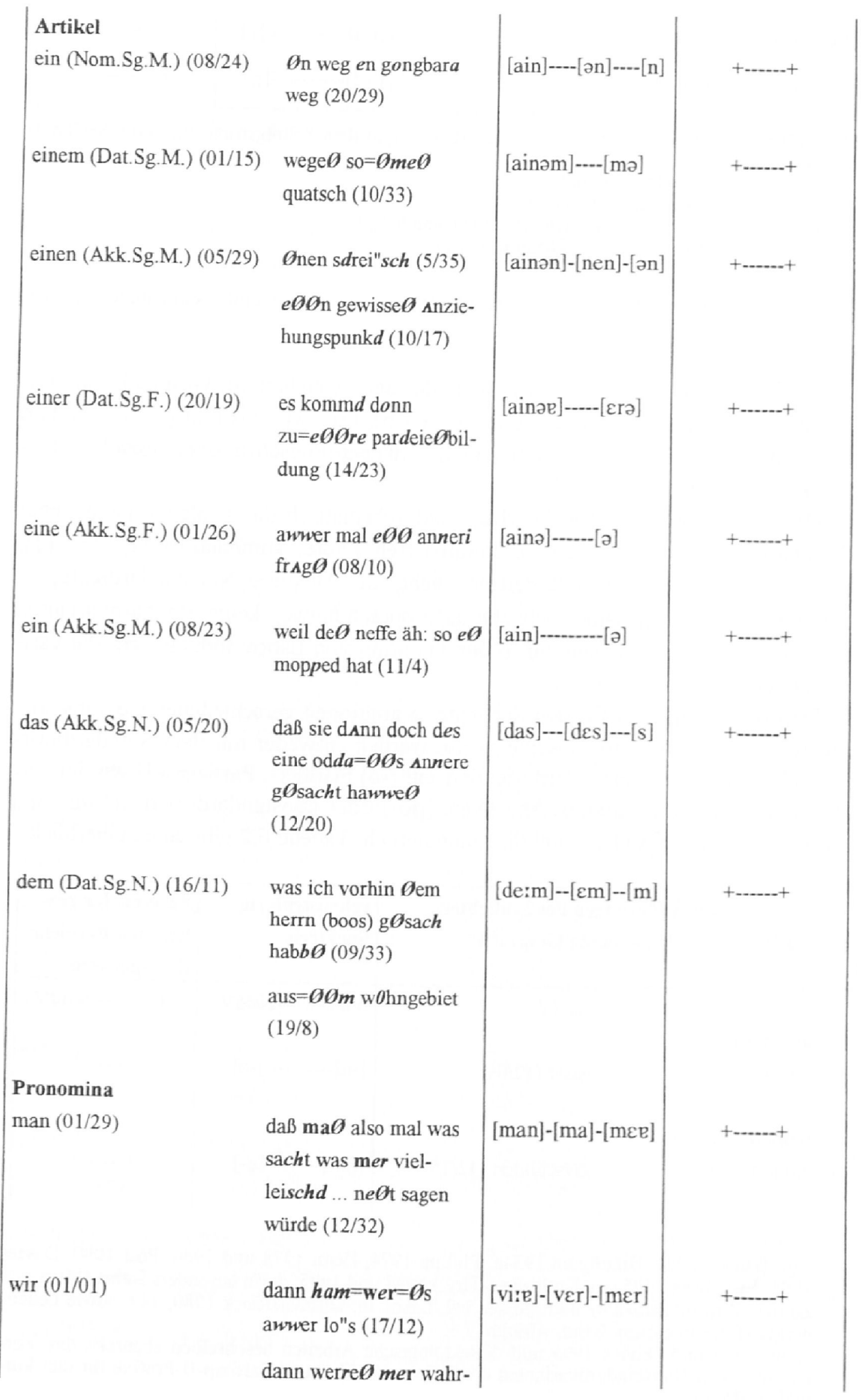




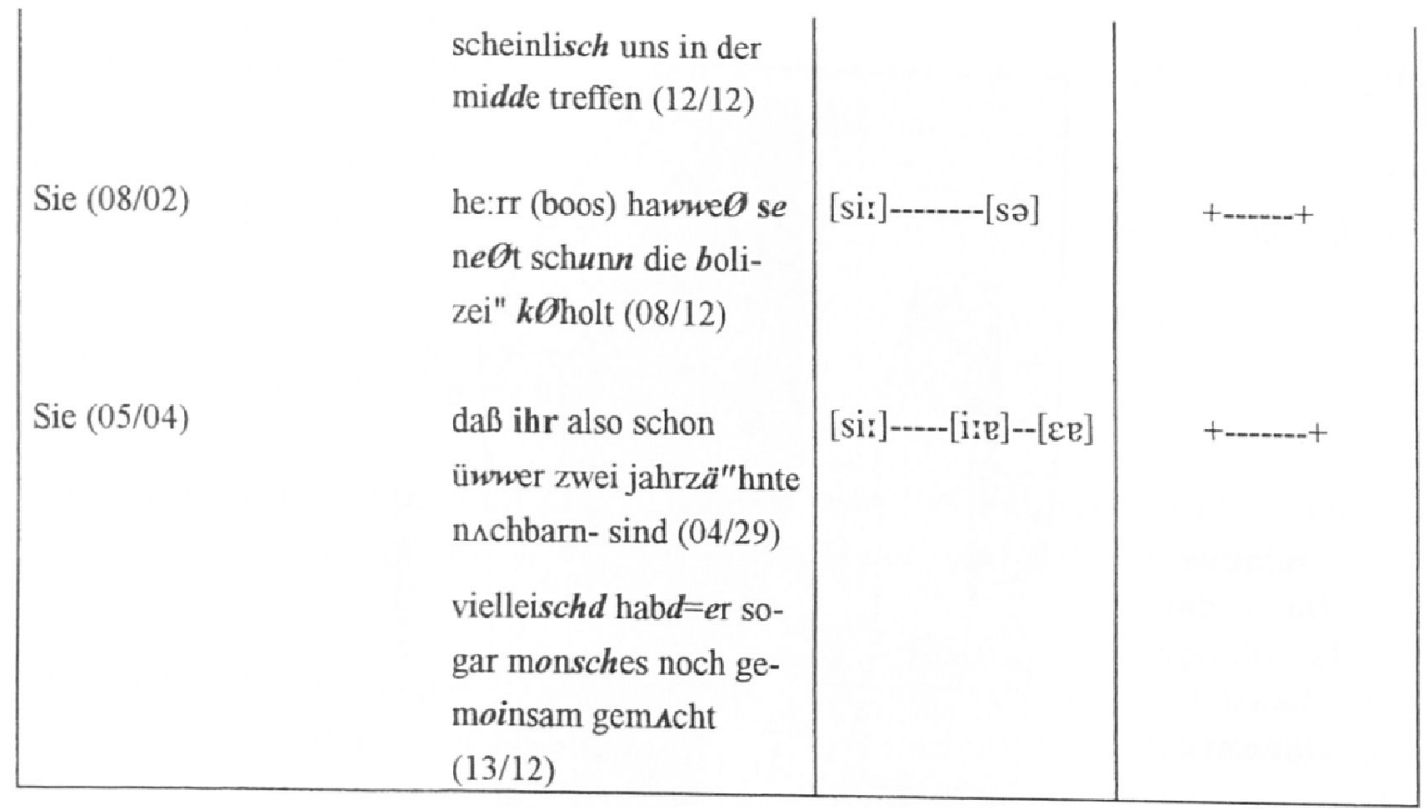

Die Ziffer vor dem Schrägstrich gibt jeweils die Seite, die Ziffer nach dem Schrägstrich die Zeile an

Nonstandardvariante

* für den Schlichter im Gespräch nicht belegbare Variante

nur die Nonstandardvarianten sind belegt

$+--+\quad$ das gesamte Spektrum ist belegt

Tab. 6.2: Morphemspektren des Schlichters generalisiert über das gesamte Gespräch

Die schematische Darstellung in der dritten Spalte illustriert, daß der Schlichter zu allen Morphemen auch die Standardvariante verwendet. Nach der getroffenen Festlegung funktionieren also die Nonstandardmorpheme als Marker.

\section{Dichte von Nonstandardmustern}

Für die vorliegende Studie greifen wir aus dem Gespräch zwei Handlungskonstellationen heraus, die in unseren Dichterechnungen weit auseinander liegen.

In einer ersten quantifizierten Beschreibung, aus der das Diagramm 7.1 resultiert, wurde für jede Handlungskonstellation der Mittelwert der Nonstandardmerkmale pro Zeile des Transkriptes errechnet. Die Handlungskonstellation „Konfliktaushandlung“ ist doppelt so lang (erstreckt sich über doppelt so viele Textzeilen) wie die Handlungskonstellation „Herstellung der Schlichtungssituation". In der ersten Handlungskonstellation finden sich im Schnitt 3,6 Nonstandardmerkmale pro Zeile, in der zweiten 6,4 (vgl. Achse: Häufigkeit). Daß die Dichten innerhalb der Handlungskonstellationen variieren, erklären wir erst in weiteren Schritten. $^{22}$

22 Vgl. Henn-Memmesheimer 1997. 


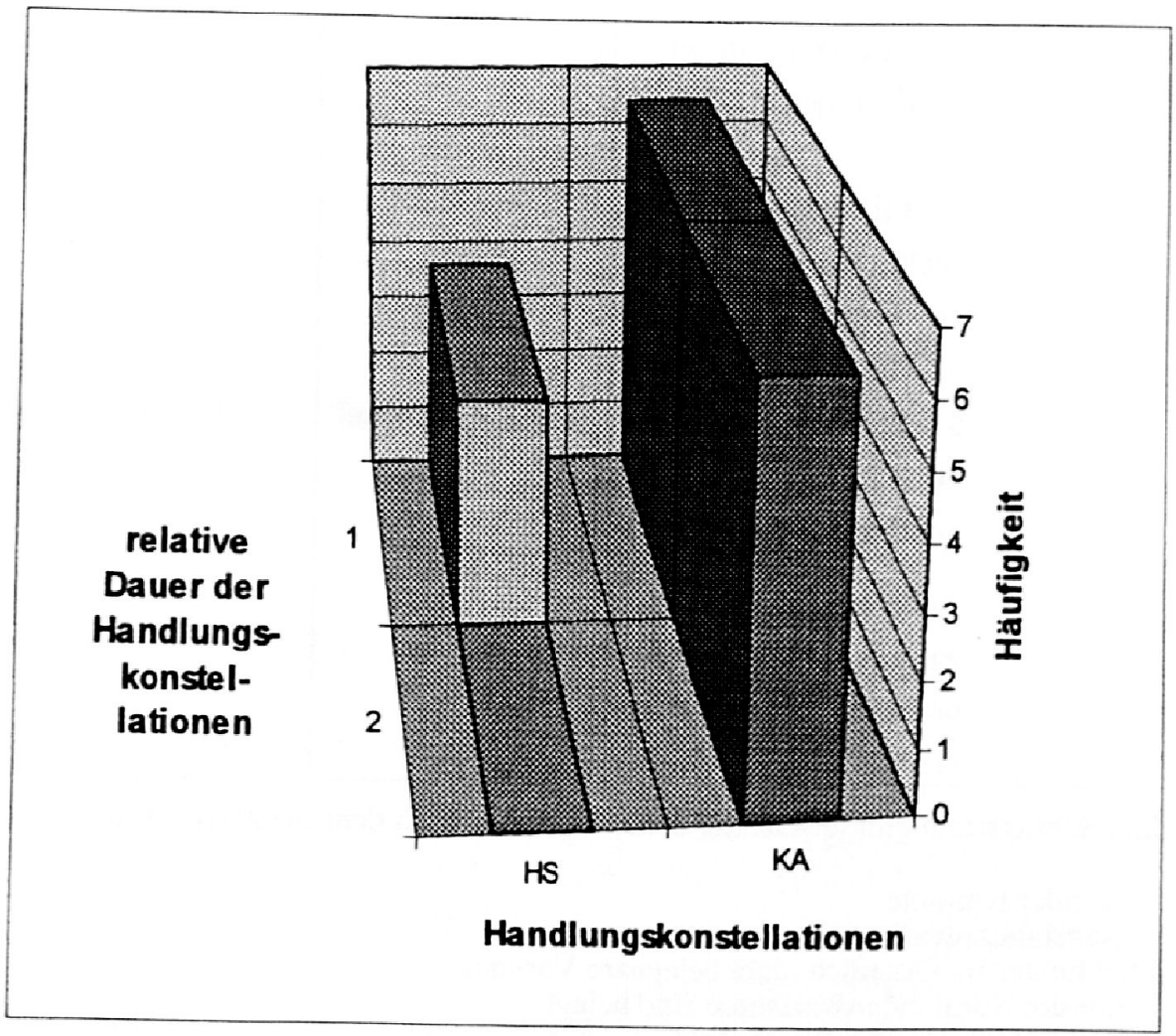

HS = Herstellung der Schlichtungssituation $\mathrm{KA}=$ Konfliktaushandlung

Diagramm 7.1: Häufigkeit der vom Schlichter verwendeten Nonstandardmuster in den beiden Handlungskonstellationen

\section{Inhaltliche Analyse und Analyse der Nonstandardverwendungen}

\subsection{Handlungskonstellation I: Herstellung der Schlichtungssituation}

Die erste Handlungskonstellation umfaßt die Eröffnung des Schlichtungsgespräches und die Erläuterung seiner Funktion in Abgrenzung zu einer Gerichtsverhandlung.

\begin{tabular}{|l|l|l|}
\hline Handlungsschritte & Seite/Zeile & Inhaltliche Beschreibung \\
\hline Eröffnung & $1 / 1-8$ & $\begin{array}{l}\text { C eröffnet durch die Vorstellung der anwesenden } \\
\text { Personen die Vergleichsverhandlung. }\end{array}$ \\
\hline Funktionserläuterung & $1 / 9-2 / 3$ & $\begin{array}{l}\text { C erläutert in Abgrenzung zum Gericht die Funk- } \\
\text { tionen einer Vergleichsbehörde. Innerhalb dieser } \\
\text { Erläuterung hebt er }\end{array}$ \\
\hline
\end{tabular}




\begin{tabular}{|l|l|l|}
\hline $\begin{array}{l}\text { Funktionserläuterung } \\
\text { (Forts.) }\end{array}$ & $1 / 20-25$ & $\begin{array}{l}\text { zum einen die Notwendigkeit einer langfristigen } \\
\text { Konfliktlösung und }\end{array}$ \\
\hline $\begin{array}{l}\text { Funktionserläuterung } \\
\text { (Forts.) }\end{array}$ & $1 / 26-2 / 3$ & $\begin{array}{l}\text { zum anderen die Klärung der Kostenregelung be- } \\
\text { sonders hervor. }\end{array}$ \\
\hline
\end{tabular}

Tab. 8.1.1: Beschreibung der Handlungskonstellation I: „Herstellung der Schlichtungssituation"

Die Handlungskonstellation I „Herstellung der Schlichtungssituation“ zeigt ein Vorkommen von Standard- bzw. Nonstandardlauten aus folgenden Spektren:

\begin{tabular}{|c|c|c|}
\hline $\begin{array}{l}\text { Beispiele aus Äußerungen des Schlichters in } \\
\text { Handlungskonstellation Intentionalität }\end{array}$ & $\begin{array}{l}\text { aus dem gesam- } \\
\text { ten Gespräch } \\
\text { rekonstruierte } \\
\text { Spektren }\end{array}$ & $\begin{array}{l}\text { Schema der in } \\
\text { Handlungskon- } \\
\text { stellation I reali- } \\
\text { sierten Bereiche } \\
\text { der Spektren } \\
\end{array}$ \\
\hline StdV-NonstdV & StdV-ב-_-NstdV & StdV---NstdV \\
\hline \multicolumn{3}{|l|}{ Indikatoren (nach Tab. 6.2) } \\
\hline$[/]-[\operatorname{sint}](01 / 4)$ & 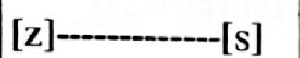 & \\
\hline [/]-_[IJ](1/01) & {$[c ̧]---[\varsigma]----\left[\int\right]$} & \\
\hline \multicolumn{3}{|l|}{ Marker (nach Tab. 6.2) } \\
\hline [-ig-](-01/20)-_-_---if-](01/29) & {$[g / k]-\cdots\left[\int\right]$} & $+-\ldots+-+$ \\
\hline$[\mathrm{dan}](01 / 23)---[\mathrm{d} \wedge \mathrm{n}](01 / 20)$ & {$[a]-\cdots[\Lambda$} & $+\cdots+$ \\
\hline$[$ ain-](01/15)-- $[$ in $](01 / 15)$ & [ai]----- $[\Lambda \mathrm{i}]$ & +--++ \\
\hline$[\operatorname{aux}](01 / 12)-[\Lambda u x](01 / 07)$ & {$[a u]--\cdots---[\Lambda u]$} & +-----+ \\
\hline [Ist](1/8)-_isd](1/25) & {$[t]$} & +-----+ \\
\hline [sint ](01/01)--------[sin](01/27) & {$[t] \ldots-\ldots[0]$} & +-----+ \\
\hline [/]-_pradogol-] $[(01 / 08)$ & {$[k]-\cdots---[g]$} & ------+ \\
\hline$[/] \cdots-\left[e: e \int d\right]$ & {$[s]-\ldots-\ldots$} & \\
\hline
\end{tabular}

Tab. 8. 1.2: Phonetische Indikatoren und Marker des Schlichters in Handlungskonstellation I

Der Schlichter realisiert in Handlungskonstellation I neben den fur das ganze Schlichtungsgespräch beschriebenen Indikatoren nur einen kleinen Teil seines Repertoires an Nonstandardlauten (vgl. Tab. 6.1). Die Tabelle gibt furr jeden ein Beispiel. Zuerst sind die Varianten aufgelistet, zu denen in der Handlungskonstellation die standardsprachliche Entsprechung vorkommt: Die in der Tabelle angewendete morphemorientierte Auflistung weist z.B. -ig- als [-ig-] und als [-if-] aus, die Belege dazu mit erweitertem Kontext sind beschäftigen (01/20) mit [-ig-], beteiligt $(01 / 29)$ mit [-if-]. 
Darüber hinaus sind Nonstandardmuster belegt, zu denen keine Standardvarianten vorkommen. Sie sind hier sehr selten und ergeben sich aus der konsequenten Anwendung des Morphembezugs, der anstelle der noch ausstehenden Umgebungsanalyse gewählt wurde. In allen Morphemen, die im Standard auslautendes [t] haben, variiert [d] mit [t]. Dies wird in der Tabelle am Beispiel von [ist]---[isd] erfaßt. Das Morphem erst erscheint nur in der Variante mit [Jd]. Das Morphem Protokoll kommt nur in der Variante mit [g] vor, in allen anderen Morphemen dieser Passage mit standard $[\mathrm{k}]$ wird $[\mathrm{k}]$ realisiert.

An morphologischen Markern werden in dieser Handlungskonstellation nur [sin] $\mathrm{zu}$ sind und eine Variante des Pronomens man eingesetzt:

\begin{tabular}{|c|c|c|}
\hline $\begin{array}{l}\text { Beispiele aus Äußerungen des Schlichters in } \\
\text { Handlungskonstellation I }\end{array}$ & \begin{tabular}{|l|} 
Aus dem gesamten \\
Gespräch rekon- \\
struierte \\
Spektren
\end{tabular} & $\begin{array}{l}\text { Schema der in } \\
\text { Handlungskon- } \\
\text { stellation I reali- } \\
\text { sierten Bereiche } \\
\text { der Spektren } \\
\end{array}$ \\
\hline 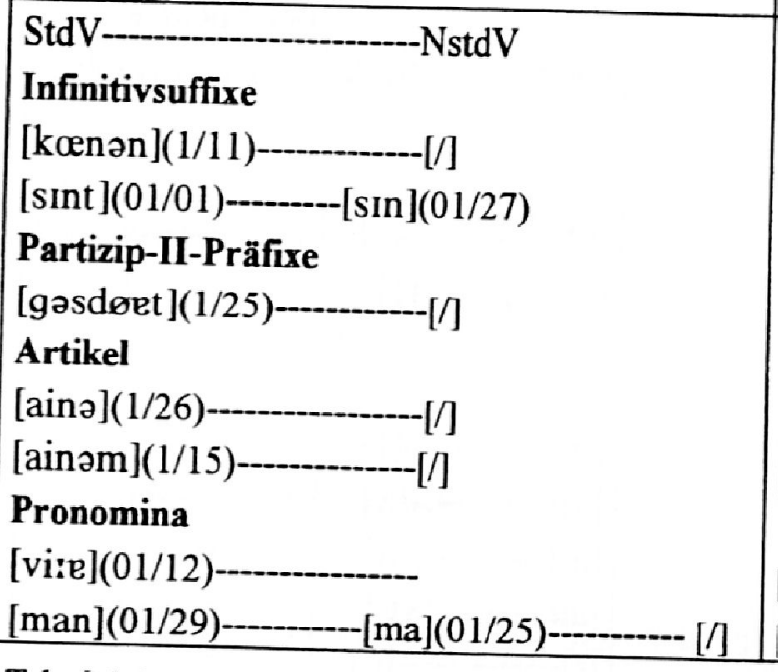 & 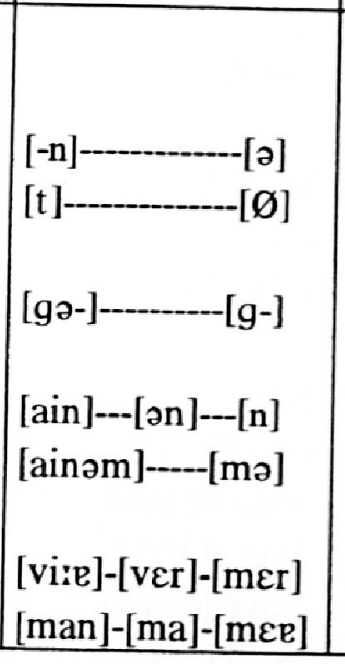 & StdV-_._--NstdV \\
\hline
\end{tabular}

Tab. 8.1.3: Morphologische Marker des Schlichters in Handlungskonstellation I

Flexionsmorpheme, Artikel und Pronomina werden also mit Ausnahme je einer Okkurrenz von [sin] und [ma] in den Standardvarianten realisiert.

\subsection{Handlungskonstellation II ,Konfliktaushandlung“}

Die zweite ausgewählte Handlungskonstellation umfaßt - bezogen auf die in Abb. 4.3 dargestellte funktionale Systematik - Teile der Konfliktaushandlung, aber auch schon Teile, die zur Herbeifürung einer Einigung gehören. Sie beginnt nach der ersten Konfliktdarstellung und -gegendarstellung. 


\begin{tabular}{|c|c|c|}
\hline Handlungsschritte & Seite/Zeile & Inhaltliche Beschreibung \\
\hline $\begin{array}{l}\text { Konfliktformulierungs- } \\
\text { angebot }\end{array}$ & $12 / 2-24$ & $\begin{array}{l}\text { Zur Durchsetzung seiner Konfliktformulierung } \\
\text { erläutert } \mathrm{C}^{23} \text { seine individuelle Vorgehensweise, } \\
\text { um eine Konfliktformulierung ausfindig zu ma- } \\
\text { chen, die beiden Parteien gerecht wird. Zur Be- } \\
\text { kräftigung seines Angebots bettet er einen Hin- } \\
\text { weis auf seine Lebenserfahrung ein, um dann } \\
\text { konkret sein Konfliktformulierungsangebot zu } \\
\text { formulieren. }\end{array}$ \\
\hline $\begin{array}{l}\text { Konfliktgegendarstel- } \\
\text { lung }\end{array}$ & $12 / 23-29$ & $\begin{array}{l}\text { B lehnt die angebotene Konfliktformulierung von } \\
\mathrm{C} \mathrm{ab} \text {. }\end{array}$ \\
\hline $\begin{array}{l}\text { Konfliktformulierungs- } \\
\text { angebot }\end{array}$ & $\begin{array}{l}12 / 28 \\
13 / 2\end{array}$ & $\begin{array}{l}\text { C bekräftigt Bs Konfliktgegendarstellung, ver- } \\
\text { sucht jedoch weiterhin seine Konfliktformulierung } \\
\text { durchzusetzen, in dem er B erneut ein Angebot } \\
\text { unterbreitet. }\end{array}$ \\
\hline $\begin{array}{l}\text { Konfliktgegendarstel- } \\
\text { lung }\end{array}$ & $13 / 1-3$ & $\begin{array}{l}\text { B räumt die Möglichkeit ein, daß das Konflikt- } \\
\text { formulierungsangebot von } C \text { zutreffen könnte, } \\
\text { entzieht sich jedoch aufgrund mangelnden Erinne- } \\
\text { rungsvermögens einer endgültigen Zustimmung. }\end{array}$ \\
\hline $\begin{array}{l}\text { Sicherstellung der Kon- } \\
\text { fliktgegendarstellung }\end{array}$ & $13 / 4-7$ & $\begin{array}{l}\text { C bewertet dieses Verhalten von B negativ, geht in } \\
\text { diesem Zusammenhang jedoch nicht näher darauf } \\
\text { ein. }\end{array}$ \\
\hline $\begin{array}{l}\text { Aufzeigen bisheriger } \\
\text { Gemeinsamkeiten }\end{array}$ & $13 / 8-14 / 4$ & $\begin{array}{l}\text { C erinnert beide Konfliktparteien an ihre frühere } \\
\text { gute Nachbarschaft im Siedlungsgebiet und ver- } \\
\text { weist hierbei auf seine lokale Ortskenntnis, seine } \\
\text { Lebenserfahrung }\end{array}$ \\
\hline $\begin{array}{l}\text { Aufzeigen langfristiger } \\
\text { Konfliktlösung }\end{array}$ & $14 / 5-7$ & $\begin{array}{l}\text { sowie die Wichtigkeit einer langfristigen Konflikt- } \\
\text { lösung, um seine Argumentation im Konfliktfor- } \\
\text { mulierungsangebot stärker bekräftigen zu können. }\end{array}$ \\
\hline
\end{tabular}

Tab. 8.2.1: Beschreibung der Handlungskonstellation II „Konfliktaushandlung“

$\overline{23}$ Zur Erläuterung: $\mathrm{C}=$ Schlichter, $\mathrm{B}=$ Antragsgegner. 
Folgende Lautspektren lassen sich belegen:

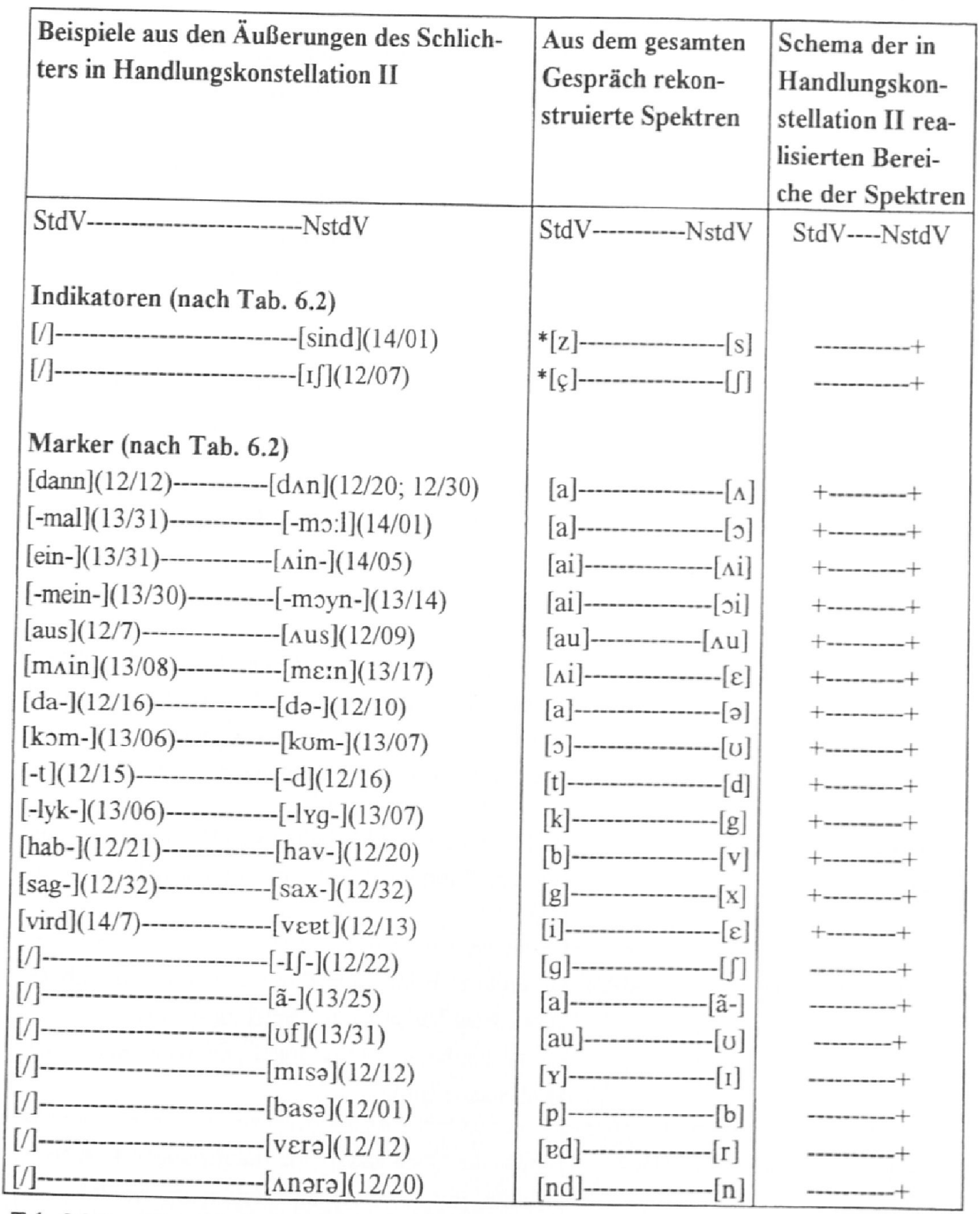

Tab. 8.2.2: Phonetische Indikatoren und Marker des Schlichters in der Handlungskonstellation
II „Konfliktaushandlung ${ }^{“}$

Der Sprecher realisiert in dieser Handlungskonstellation deutlich mehr Nonstandardmuster aus seinem Repertoire als in der ersten Handlungskonstellation. Daß dies nicht allein der Länge der Handlungskonstellation geschuldet ist, zeigt sich schon in der Tabelle: zu vielen Nonstandardvarianten erscheint keine Standardvari- 
ante, obwohl diese im Blick auf das gesamte Gespräch (siehe Tab. 6.2) belegbar sind.

Im Bereich der morphologischen Varianz ergibt sich ein ähnliches Bild:

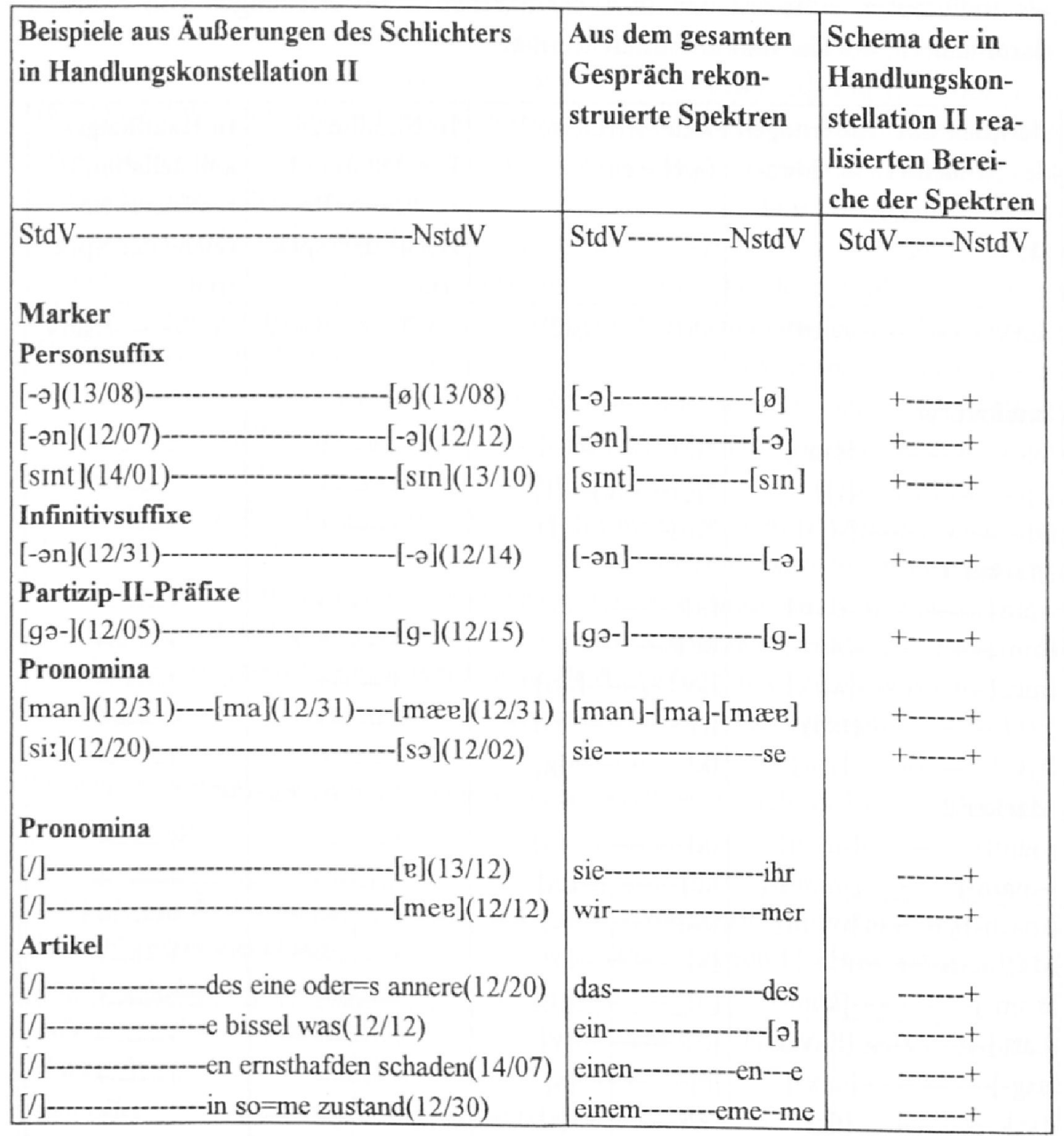

Tab. 8.2.3: Morphologische Marker des Schlichters in Handlungskonstellation II

Es gibt hier eine Reihe von Spektren, aus denen nur die Nonstandardmuster und keine Standardmuster realisiert werden. 
9. Indikatoren, verschiedene Typen von Markern und Situationsstrukturierung

Die folgende Tabelle gibt einen Überblick über Nonstandardmuster, die invariant als Indikatoren aufteten und über charakteristische Verteilungen von Nonstandardmustern, die als Marker variiert werden:

\begin{tabular}{|c|c|c|c|}
\hline $\begin{array}{l}\text { Beispiele aus Äußerungen } \\
\text { des Schlichters in Hand- } \\
\text { lungskonstellation I und } \\
\text { II }\end{array}$ & $\begin{array}{l}\text { Rekonstruierte } \\
\text { Spektren }\end{array}$ & $\begin{array}{l}\text { In Handlungs- } \\
\text { konstellation I } \\
\text { realisierte Be- } \\
\text { reiche der Spek- } \\
\text { tren }\end{array}$ & $\begin{array}{l}\text { In Handlungs- } \\
\text { konstellation II } \\
\text { realisierte Be- } \\
\text { reiche der Spek- } \\
\text { tren } \\
\end{array}$ \\
\hline 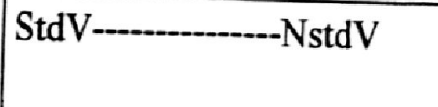 & StdV-.----NstdV & StdV----NstdV & StdV------NstdV \\
\hline Indikatoren & & & \\
\hline [/]-_-_sint] & *[z]- & …..-+ & -.---+ \\
\hline$[/]$ & *[ç]-----[६]---[J] & --o-+ & -..--+ \\
\hline Marker 1 & 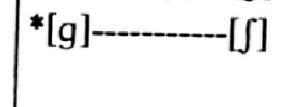 & -...-.+ & \\
\hline [dan]-_- $\left[d_{\Lambda} n\right]$ & {$[\mathrm{a}]$} & $+\ldots+$ & $+\ldots+$ \\
\hline [ain-]-_ [nin-] & 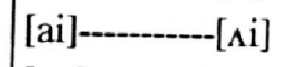 & +-..-- & +-...-+ \\
\hline$[a u x]$ & 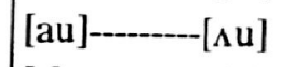 & +.--..- & +...--+ \\
\hline [Ist ]__-_ [Isd] & {$[t] \ldots[d]$} & $+\cdots+$ & $+\ldots+$ \\
\hline [lyk-]_-___-_lyg-] & {$[k]-\ldots[-\ldots$} & $\ldots+$ & $+\cdots$ \\
\hline $\begin{array}{l}\text { Marker 2 } \\
\text { [-ma:l]--_-_-mo:l] }\end{array}$ & [a]-_a] & + & $+\ldots+$ \\
\hline [-main-]-_-_-_-moin-] & [ai]_-_....--[oi] & + & $+\ldots+$ \\
\hline [msin-]-_-_me:n] & {$[\mathrm{Ai}] \ldots[\varepsilon]$} & $+\ldots$ & $+\ldots+$ \\
\hline 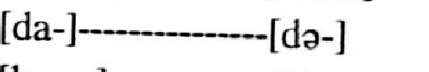 & [a]-_._. & + & +--- + \\
\hline$[\mathrm{k} э \mathrm{~m}-]$ & [a] & +- & +- \\
\hline [hab-]_._._[hav-] & $[b] \ldots-\ldots v]$ & +- & +---++ \\
\hline [sag-] [sax-] & [g] & + & +--.+- \\
\hline [fyr]__._-_fir] & {$[\mathrm{Y}]$ - } & $+\cdots$ & $\ldots+\cdots+$ \\
\hline Marker 3 & & & \\
\hline & [a]-_-_ã-] & +- & \\
\hline $\begin{array}{l}\text { [auf-]-_-_-_uf-] } \\
\text { [virt]-_[vert] }\end{array}$ & & $\begin{array}{l}+-1 . \\
+\cdots-\infty\end{array}$ & \\
\hline
\end{tabular}

Tab. 9.1: Phonetische Indikatoren und Marker in Handlungskonstellation I und II

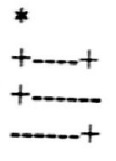

für den Schlichter nicht belegbare Variante

das gesamte Spektrum ist belegt

nur die Standardvariante ist belegt

nur die Nonstandardvarianten sind belegt 
Indikatoren sind Nonstandardmuster, die für den Sprecher offenbar unvermeidbar sind: Sie kommen in beiden Konstellationen vor.

Marker 1 sind Nonstandardmuster, die sich ebenfalls in beiden Handlungskonstellationen finden. Im Gegensatz zu den Indikatoren sind sie aber vermeidbar. Ihre Variation kann nicht auf der Ebene der situativen Handlungskonstellationen, sondern nur auf anderen Ebenen, etwa durch Analyse von Formulierungen und Reformulierungen erfaßt werden. Illustrativ ist das Beispiel mit [a] und [ $\mathrm{\Lambda}$ : unn wenn des erschd vor gerischd kommt äh dann verhärden sisch ja die fronden in aller regel ja so daß dAnn das zusammenleben doch ernsthaft gesdört ${ }^{24}$ iss (01/23-24, in literarischer Umschrift).

Marker 2 sind Nonstandardmuster, die nur in nonstandardorientierten Handlungskonstellationen vorkommen, dort allerdings auch wieder im Wechsel mit der Standardvariante. Im folgenden Beispiel aus Handlungskonstellation II erscheinen Varianten aus den Bereichen [a] und [0] eines Spektrums nebeneinander in einer Formulierung und ihrer expandierenden Reformulierung: unn jetzt uf einmal jetzd wo die kinda größer sind äh: wo se hald eØmol eØ eigenständisches leweØ entwiggeln (13/31-14/02, in literarischer Umschrift).

Marker 3 sind Nonstandardmuster mit der profiliertesten Verteilung. Die Standardmuster dieser Spektren finden sich nur in Handlungskonstellationen mit geringster Dichte von Nonstandardelementen, die Nonstandardmuster ausschließlich in Umgebungen mit großer Dichte von Nonstandardelementen. Marker 3 sind z.B. Nasalierungen: awwer isch habbØ se neØt ã'ØgØsdegd (13/25), wird in Handlungskonstellation II formuliert, im Aufzeigen von Gemeinsamkeiten.

Dieselben verteilungsbedingten Markertypen lassen sich auch im Bereich der Morphologie zeigen:

\begin{tabular}{|c|c|c|c|}
\hline $\begin{array}{l}\text { Beispiele aus Äußerungen } \\
\text { des Schlichters aus Hand- } \\
\text { lungskonstellation I und II }\end{array}$ & \begin{tabular}{|l} 
Variation von \\
\end{tabular} & $\begin{array}{l}\text { Handlungs- } \\
\text { konstellation I }\end{array}$ & $\begin{array}{l}\text { Handlungs- } \\
\text { konstellation II }\end{array}$ \\
\hline 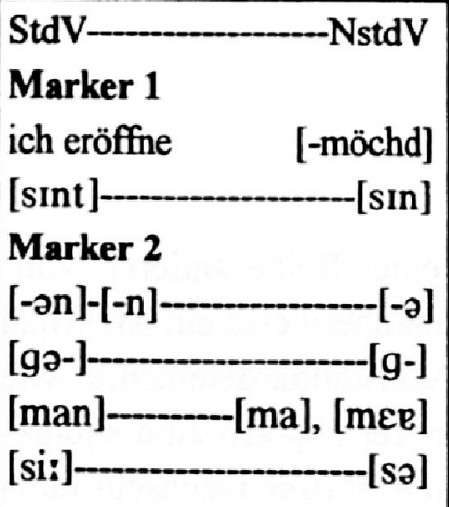 & $\begin{array}{l}\text { Personalsuffix -ə } \\
\text { Infinitivsuffix } \\
\text { Partizip II-Suffix } \\
\text { Pronomina }\end{array}$ & 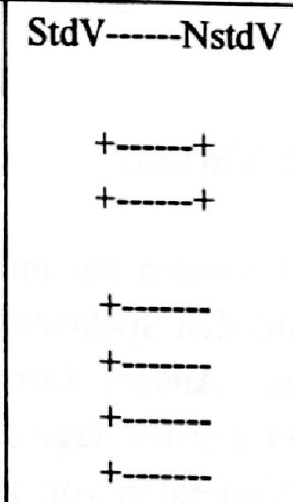 & 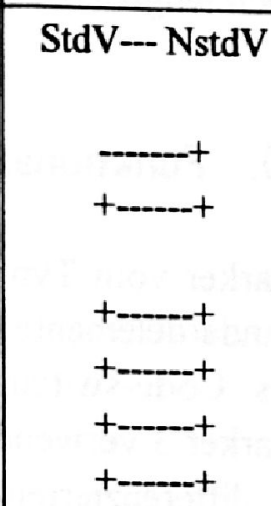 \\
\hline
\end{tabular}

$\overline{24} s d$ ist von der Standardorthographie st abgeleitet und am Wortanfang als [ $\left.\int \mathrm{d}\right] \mathrm{zu}$ lesen. 


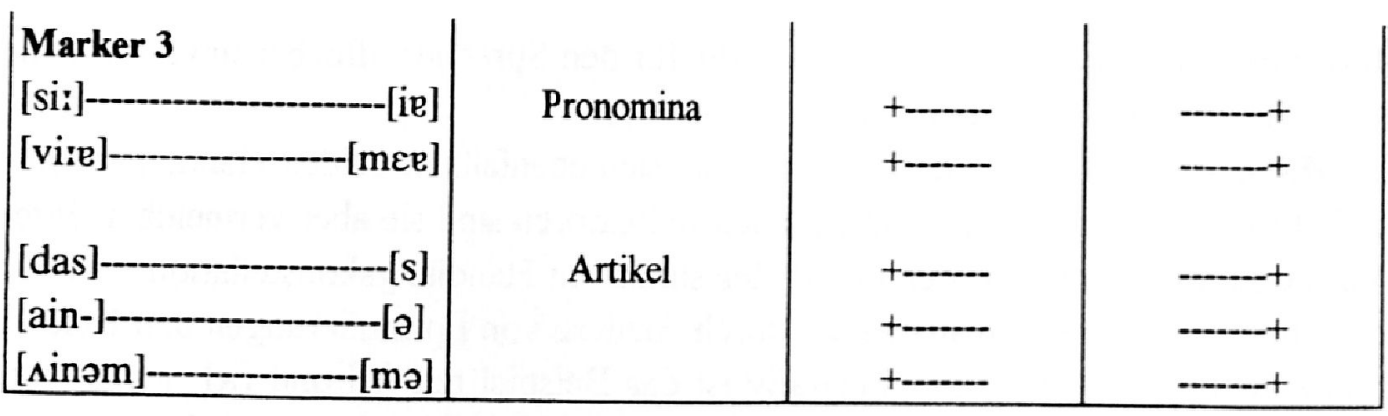

Tab. 9.2: Morphologische Marker in Handlungskonstellation I und II *

$\begin{array}{ll} & \text { für den Schlichter nicht belegbare Variante } \\ \text { das gesamte Spektrum ist belegt } \\ +--+ & \text { nur die Standardvariante ist belegt } \\ +-+ & \text { nur die Nonstandardvarianten sind belegt }\end{array}$

Marker 1 werden selbst in der standardorientierten Handlungskonstellation variiert, die Standardvariante ist selten.

Marker 2 sind an die nonstandardorientierte Handlungskonstellation gebunden: im Konfliktformulierungsangebot in Handlungskonstellation II häufen sich die Nonstandardmuster: sie hawweØ mir des awwer vorhin so schön gØschildert, auch so plasdisch un drasdisch gØschilderd - (S.12/16). Die Wahl der Standardvariante zehn Zeilen zuvor ist der formellere Einstieg in diese Passage: sie haben mir ja vorhiin- * geschildert- (S.12/5).

Marker 3 sind extreme Markierungen, die noch konsequenter als die übrigen morphologischen Marker an der Strukturierung der Situation beteiligt sind: Im zweiten Konfliktformulierungsangebot (vgl. Tab. 8.2.1, 3. Zeile), wo der Schlichter eine eigene Konfliktformulierung durchsetzen, aber auch eine Version von B stabilisieren muß, erscheint die Nonstandardvariante [mo] von einem: ja unn_kann= $\emptyset \mathrm{s}$ net gØradØ in_ so=ØmeØ zusdAnd dAnn erschd reschd bassieren daß man über die sdränge schlägd (12/30). In der Handlungskonstellation I heißt es in einer Umgebung mit lediglich Indikatoren: $d a \beta$ wir versuchen soll/ sollen in solschen- * dingen in solschen fällen mit einem rescht/ kriminellen unreschdsgehalt äh:- ** nach möglischkeid diese dinge vom gerischd fernzuhalden (1/15).

\section{Funktionale Varianz}

Marker vom Typ 3 werden nur im Zusammenhang mit einer Reihe anderer Nonstandardelemente aus den Spektren verwendet. Dies ist üblicherweise ein Merkmal des Code-switching. Anders formuliert: Wenn alle Nonstandardelemente wie Marker 3 verwendet würden, läge die klassische Situation der objektiv und subjektiv differenzierten Varietäten vor, zwischen denen man nur abrupt wechseln kann und zwischen denen man unter wohldefinierten Bedingungen wechseln muß. Gumperz 1994 kommt bei seiner Analyse des Materials aus dem Mannheimer Stadtsprachenprojekt zu einem solchen Ergebnis. Die Wahl von Standardvarianten 
stehe hier im Kontext „offiziellen Sprechens“. Diese durch die Vorgabe eines Standards legitimierte Verteilung ist sicher in allen Analysen zu berücksichtigen, scheint aber bezogen auf unser Material zu pauschal.

Andererseits formulierte gerade Gumperz „Discourse Strategies“ und näherte sich damit einem handlungsbasierten Variationsbegriff, der vielseitiger ist als eine Analyse von Domänenverteilungen. Das vorliegende Schlichtungsgespräch mit den ständigen Bewegungen auf einem breiten Spektrum von Standard- und Nonstandardmustern („Code-shifting') verlangt eine Analyse, die flexibel genug ist, situative Handlungsspielräume zu beschreiben. In einem ersten Ansatz haben wir hier bezogen auf den definierten Rahmen der Vergleichsverhandlung - versucht, Möglichkeiten der Situationsstrukturierung durch Auswahl von sprachlichen Mustern mit entsprechenden Signifikanzen zu umreißen. Darüber hinaus zeichnen sich signifikante Varianzen innerhalb der Handlungskonstellationen ab, die wir auf anderen Ebenen analysieren: z.B. themenabhängiges, argumentationsabhängiges und formulierungsabhängiges Variieren. 